'Servicio de Urgencias Hospital Universitario La Paz, Madrid, España.

${ }^{2}$ Servicio de Medicina Interna Hospital Universitario La Paz, Madrid, España. ${ }^{\mathrm{a} P h D .}$

Para el desarrollo del estudio y escritura del manuscrito no se ha recibido financiación. Todos los autores declaran no tener conflicto de intereses.

Recibido el 28 de julio de 2014 aceptado el 25 de agosto de 2015.

Correspondencia a: M. Angélica Rivera N. Calle Maestro Guerrero $2-1^{\circ} \mathrm{A}$. CP 28015. Madrid. España. mangelicariveran@hotmail.com

\section{Motivos de consulta de pacientes con enfermedades autoinmunes sistémicas en el servicio de urgencias de un hospital terciario}

\section{MARÍAANGÉLICA RIVERA NÚÑEZ ${ }^{1}$, CARLOS MARTÍNEZ CABALLERO ${ }^{1}$ MIRIAM ESTÉBANEZ MUÑOZ ${ }^{1}$, MARÍA ELENA CALVIN GARCÍA ${ }^{1}$, ÁNGEL ROBLES M.², MANUEL QUINTANA DÍAZ ${ }^{1, \mathrm{a}}$ \\ Causes for consultation in the emergency room among patients with systemic autoimmune diseases}

Background: The causes of acute decompensations of patients with systemic autoimmune diseases are not well known. Aim: To describe the causes for consultation in an emergency room of patients with systemic autoimmune diseases. Material and Methods: Review of medical records of patients with systemic autoimmune diseases, aged over 14 years, consulting in an emergency room of a general hospital during three months. Results: In the study period, 166 patients with systemic autoimmune diseases consulted in the emergency room, of a total of 18,153 consultations $(0.9 \%)$. Patients with rheumatoid arthritis were those that consulted with higher frequency (37\%) followed by patients with systemic lupus erythematosus (21\%). The most common causes for consultation were cardiovascular diseases in 25\%, followed by digestive disorders in 15\%. The most common diagnosis was chest pain with suspected ischemic heart disease in $36 \%$. No differences in cardiovascular risk factors were observed between those patients consulting for cardiovascular diseases and those consulting for other causes. Conclusions: The most common cause of consultation in the emergency room of patients with systemic autoimmune diseases is cardiovascular.

(Rev Med Chile 2015; 143: 1546-1551)

Key words: Autoimmune disease; Cardiovascular diseases; Emergency medical care; Emergency Service, hospital.
L

os pacientes con enfermedades autoinmunes sistémicas (EAS) acuden a los Servicios de Urgencias (SU) por causas muy diversas, y no siempre por complicaciones específicas de su enfermedad. Las complicaciones propias de las EAS que precisan atención urgente han sido descritas y suponen un reto diagnóstico y terapéutico para el médico de urgencias, principalmente por la heterogeneidad de su presentación y por la escasa prevalencia en la población general ${ }^{1,2}$. Sin embar- go, no está definido si estas complicaciones son la causa de descompensación aguda más frecuente de estos pacientes.

Por otro lado, los pacientes con EAS, tanto por su patología de base como por efectos secundarios de los tratamientos instaurados, pueden presentar complicaciones agudas que es necesario conocer y tratar en forma precoz por parte de los especialistas en Medicina de Urgencias y Emergencias.

La incidencia exacta de las consultas a los SU 
por parte de pacientes con EAS se desconoce, así como el grado de frecuencia de cada motivo de consulta ${ }^{1}$. En 1988, Schlosser et $\mathrm{al}^{3}$ describieron una incidencia de $9 \%$ de consultas por causas que denominó como reumatológicas, en una serie de 5.592 pacientes que consultaron a un Servicio de Urgencias general, no existiendo series similares desde entonces.

El objetivo de este artículo es describir y analizar los motivos de consulta de los pacientes con EAS en un SU general de un Hospital terciario.

\section{Pacientes y Métodos}

Estudio observacional retrospectivo unicéntrico. Se incluyeron todos los pacientes mayores de 14 años con antecedentes de EAS que consultaron en el Servicio de Urgencias del Hospital Universitario La Paz en el período comprendido entre el 1 de octubre y el 31 de diciembre de 2011.

El Hospital Universitario La Paz es un centro hospitalario público de tercer nivel, dependiente de la Comunidad de Madrid, situado en la zona norte de Madrid, con una infraestructura formada por un espacio total de casi $200.000 \mathrm{~m}^{2}$, compuesto por un entramado de 18 edificios que forman los cuatro hospitales principales: Hospital General, Hospital Maternal, Hospital Infantil, y Hospital de Traumatología y Rehabilitación. Consta de tres Servicios de Urgencias: Infantil, Maternal y de Adultos, con más de 200.000 urgencias anuales. Diariamente, el Servicio de Urgencias de Adultos atiende aproximadamente 300 urgencias médicas (no quirúrgicas) con una población asignada de aproximadamente 500.000 habitantes.

La recolección de la información se realizó en forma retrospectiva mediante la revisión de la historia clínica electrónica y los datos administrativos hospitalarios. Contamos con registro electrónico de todos los pacientes que consultan en urgencias, historia clínica electrónica y registro de todas las pruebas complementarias realizadas en toda la comunidad de Madrid.

Se recogieron de forma retrospectiva variables demográficas, tipo de EAS diagnosticada previamente, motivo de consulta al SU, presencia de comorbilidad y factores de riesgo cardiovascular (hipertensión arterial, diabetes mellitus, historia de tabaquismo, dislipemia), diagnóstico de urgencias y destino del paciente (ingreso o alta desde el SU).

Los motivos de consulta fueron definidos considerando el síntoma principal que el paciente expresó en la sala de clasificación (sala de triage) a su llegada al SU definido por el facultativo de esta área del SU.

Se definió como motivo de consulta cardiovascular: disnea, palpitaciones, síncope, presíncope y/o dolor torácico de probable origen isquémico definido por criterios clínicos de localización, irradiación y síntomas acompañantes según práctica clínica habitual y valorado en la sala de clasificación por el facultativo de triage. Como causa infecciosa: a la presencia de fiebre. Como causa digestiva: a la presencia de dolor abdominal, diarrea y/o sospecha de hemorragia digestiva. Causa musculoesquelética: a la presencia de artralgias, aumento de volumen articular, impotencia funcional y/o dolor musculoesquelético. Causa neurológica: cefalea como síntoma primario, sin el antecedente de haber presentado fiebre, o la presencia de cualquier focalidad neurológica.

Se definió como diagnóstico definitivo, al registrado en el informe de alta del SU o de los distintos Servicios Clínicos del Hospital si el paciente fue ingresado en una planta de hospitalización convencional o en la Unidad de Cuidados Intensivos.

Las variables continuas son expresadas como media (desviación estándar, DE) si seguían la distribución normal, o como mediana (rango intercuartil) si no seguían la distribución normal. Se usaron la t de Student o la U-Mann-Whitney, respectivamente. Las variables categóricas son expresadas como porcentajes, y se compararon con el test de chi-cuadrado. En el análisis univariado se introdujeron variables demográficas, comorbilidades, motivos de consulta y factores de riesgo cardiovascular recogidos.

El análisis estadístico se realizó mediante el paquete estadístico con el programa SPSS 15.0 (Inc, IL, USA). Se consideró significativo un valor de $\mathrm{p}<0,05$.

\section{Resultados}

Durante el período de estudio, se identificaron 166 pacientes entre 19 y 91 años con antecedentes de EAS que consultaron al SU, de un total de 18.153 consultas médicas de urgencias, lo que co- 
rresponde a $0,91 \%$ de todas las urgencias médicas atendidas durante el período de estudio.

La edad media fue de 62,7 $\pm 18,1$ años. De los 166 casos, $122(73,5 \%)$ fueron mujeres. No se observó diferencia estadísticamente significativa en la edad según sexo (mujeres $62,0 \pm 18,3$ años, hombres $64,4 \pm 17,5$ años; $p=0,45$ ).

Las EAS que con más frecuencia presentaron los pacientes del estudio fueron: artritis reumatoide $(61 / 166,36,7 \%)$, lupus eritematoso sistémico (35/166, 21,10\%) (Figura 1).

El motivo más frecuente de consulta en el Servicio de Urgencias fue cardiovascular (42/166, $25 \%)$, seguido de la causa infecciosa $(24 / 166$, $15 \%)$, y la digestiva $(24 / 166,15 \%)$, como se muestra en la Figura 2.
La EAS más frecuente en los pacientes que consultaron por motivo cardiovascular fue la AR $(15 / 42,35,7 \%)$. La edad media de los pacientes que consultaron por esta causa, fue de $73,2 \pm 15,3$ años. Un $67 \%$ correspondió a mujeres (28/42). Los motivos de consulta en este grupo de pacientes correspondieron a: dolor torácico con sospecha de cardiopatía isquémica $35,7 \%$ ( $15 / 42$ pacientes), disnea $40,6 \%$ (17/42 pacientes), síncope o presíncope $12 \%$ ( $5 / 42$ pacientes), crisis hipertensiva $7,2 \%$ (3/42 pacientes) y palpitaciones $2 \%(2 / 42$ pacientes).

Las características demográficas de los pacientes con EAS que acudieron al SU por causas cardiovasculares se reflejan en la Tabla 1.

Se observó diferencia estadísticamente signifi-
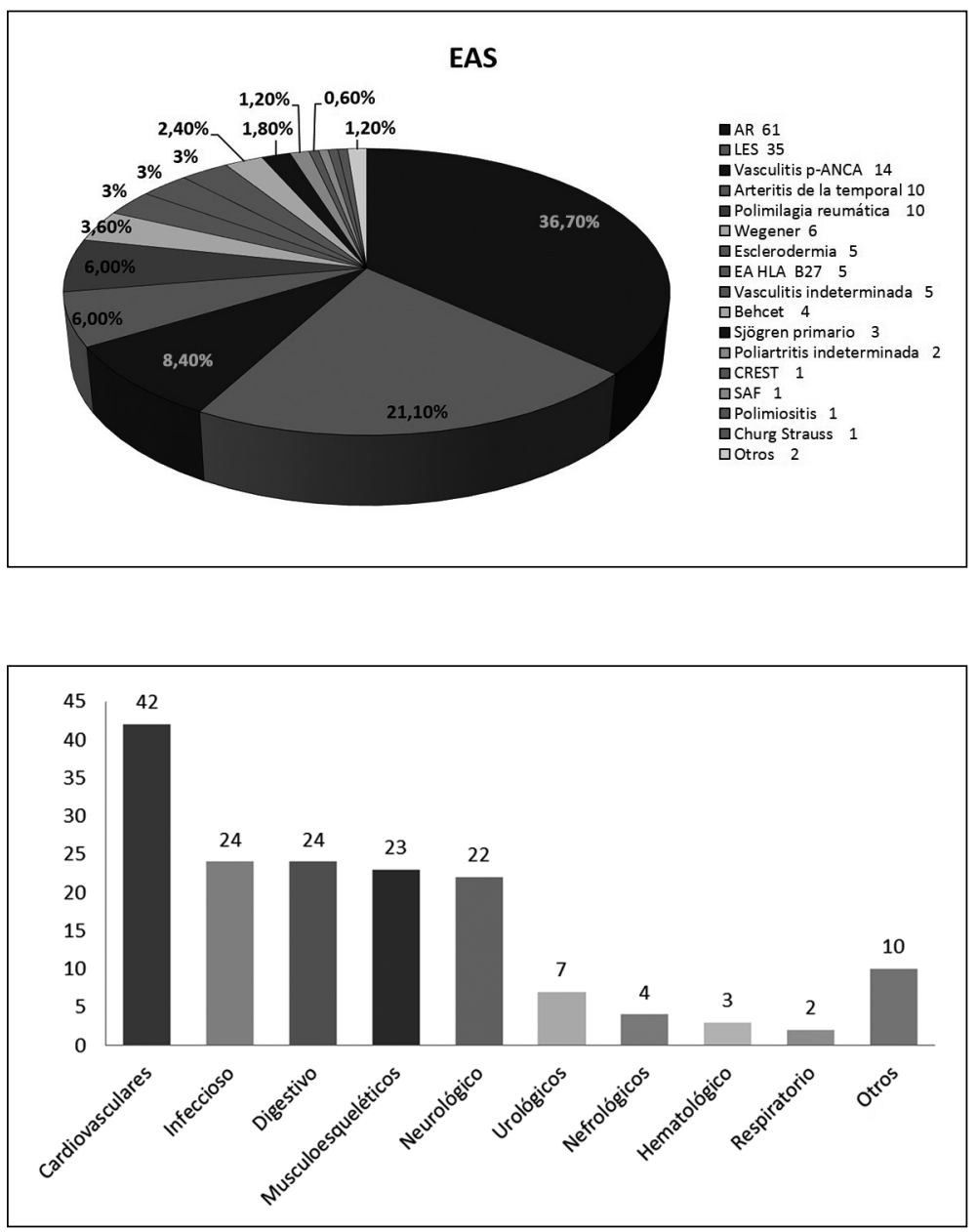

Figura 2. Motivos de consulta de los pacientes con EAS que consultan al servicio de urgencias. 
Tabla 1. Características de los pacientes con EAS que consultan al servicio de urgencias por motivos cardiovasculares

$\begin{array}{ll}\begin{array}{l}\text { Características demográficas } \\ \text { Edad media } \\ \text { Sexo }\end{array} & \begin{array}{l}73,23 \pm 15,35 \\ \text { Mujeres 28 (67\%) } \\ \text { Hombres 14 (33\%) }\end{array} \\ \begin{array}{l}\text { EAS del paciente } \\ \text { Artritis reumatoide }\end{array} & 15(35,7 \%) \\ \text { Vasculitis p ANCA } & 6(14,3 \%) \\ \text { Esclerodermia } & 5(11,9 \%) \\ \text { Polimialgia reumática } & 5(11,9 \%) \\ \text { Lupus eritematoso sistémico } & 4(9,6 \%) \\ \text { Vasculitis indeterminada } & 2(4,8 \%) \\ \text { Otros } & 5(4,8 \%) \\ \text { Motivos de consulta } & \\ \text { Dolor torácico } & 15(35,7 \%) \\ \text { Disnea } & 17(40,6 \%) \\ \text { Crisis hipertensiva } & 3(7,2 \%) \\ \text { Síncope/presíncope } & 5(12 \%) \\ \text { Palpitaciones } & 2(4,8 \%) \\ \text { Destino del paciente } & \\ \text { Alta } & 22(52,4 \%) \\ \text { Ingreso } & 20(47,6 \%)\end{array}$

cativa en la edad de los pacientes que consultaron por motivos cardiovasculares y no cardiovasculares $(73,2 \pm 15,3$ años vs $59,2 \pm 17,6$ años, $\mathrm{p}<0,001)$.

El 67,5\% (112/166) de los pacientes fueron dados de alta y $32,5 \%(54 / 166)$ requirieron ingreso. La AR correspondió a la EAS más frecuente en los pacientes que consultaron por motivos cardiovasculares, tanto en el grupo de los que requirieron ingreso $(45 \%)$ como en los que no requirieron ingreso $(27 \%)$.

De los pacientes que consultaron por motivos cardiovasculares, $47,6 \%$ requirió ingreso (20/42) sin diferencia estadísticamente significativa en la edad de los que requirieron ingreso $(77,5 \pm 11,4$ vs $69,3 \pm 17,5$ años, $\mathrm{p}=0,07)$, ni en la distribución por sexo (60\% mujeres vs $73 \%$ mujeres, $\mathrm{p}=0,05)$.

En este grupo de pacientes, los diagnósticos de alta fueron: insuficiencia cardiaca 9/20 (43,2\%) cuya etiología es la cardiopatía isquémica en $14 / 20$ pacientes; angina inestable $3 / 20(14,4 \%)$; tromboembolismo pulmonar $3 / 20(14,4 \%)$ y síncope de origen cardiogénico $1 / 20(4,8 \%)$.

No se observó diferencia en la prevalencia de los factores de riesgo cardiovascular descritos entre los pacientes que acudieron por motivo de consulta de origen cardiovascular (42/166 pacientes) y los pacientes que consultaron por otros motivos (124/166 pacientes). La distribución de los factores de riesgo cardiovascular entre estos dos grupos de pacientes se muestra en la Tabla 2.

\section{Discusión}

Este estudio describe los motivos de consulta médica de pacientes con EAS en un Servicio de Urgencias de un Hospital Terciario. Las causas de índole cardiovascular han sido el principal motivo de consulta, siendo más frecuente que las causas directamente relacionadas con las EAS. Esto difiere del resultado de estudios previos, si bien la gran mayoría de ellos se han realizado sobre cohortes de pacientes con diagnóstico de lupus eritematoso sistémico (LES) ${ }^{4,5}$ o en unidades de consultas reumatológicas ${ }^{6}$ y no en Servicios de Urgencias Generales y con objetivos distintos a los de nuestro estudio: estudio observacional descriptivo, de la frecuencia y motivos de consulta de los pacientes con EAS, en un Servicio de Urgencias General ${ }^{1,2}$.

Los hallazgos de nuestro estudio podrían explicarse en parte por la alta edad media de los

Tabla 2. Prevalencia de los factores de riesgo cardiovascular de los pacientes con EAS que consultaron al servicio de urgencias en función del tipo de consulta

\begin{tabular}{|lcccc|}
\hline $\begin{array}{l}\text { Factor de riesgo } \\
\text { cardiovascular }\end{array}$ & $\begin{array}{c}\text { Total } \\
(\mathbf{n = 1 6 6 )}\end{array}$ & $\begin{array}{c}\text { Motivo de consulta } \\
\text { cardiovascular }(\mathbf{n = 4 2 )}\end{array}$ & $\begin{array}{c}\text { Motivo de consulta no } \\
\text { cardiovascular (n= 124) }\end{array}$ & p \\
\hline Hipertensión arterial & $78(46,9 \%)$ & $19(45,2 \%)$ & $59(47,5 \%)$ & 0,142 \\
\hline Diabetes mellitus & $32(19,2 \%)$ & $9(21,4 \%)$ & $23(18,5 \%)$ & 0,291 \\
\hline Tabaquismo & $28(22,5 \%)$ & $7(16,6 \%)$ & $21(16,9 \%)$ & 0,780 \\
\hline Dislipemia & $28(16,8 \%)$ & $7(16,6 \%)$ & $21(16,9 \%)$ & 0,780 \\
\hline
\end{tabular}


pacientes incluidos y por la presencia de factores de riesgo cardiovascular, sin embargo, no hay estudios que definan la prevalencia de estos factores de riesgo cardiovascular clásicos en una cohorte general de pacientes con EAS.

Se sabe que la arterioesclerosis precoz y la enfermedad cardiovascular es una causa importante de morbilidad y mortalidad más precoz en este grupo de pacientes y que depende de factores como la inflamación crónica, la duración y actividad de la EAS y la terapia inmunosupresora. Urowitz et $\mathrm{al}^{7}$ describieron un patrón bimodal de mortalidad en pacientes con LES, diferenciando un primer grupo de pacientes con muerte precoz desde el diagnóstico de LES, y un segundo grupo de muerte tardía. En el primer grupo, la causa de la muerte estaba directamente relacionada con el LES, y en el segundo grupo, la causa más frecuente de muerte se debía a enfermedad cardiovascular. Por tanto, cabe suponer que el perfil de la mayoría de nuestros pacientes podrían corresponder a los que Urowitz et al incluyen en el segundo grupo. Al igual que en el LES, en los pacientes con artritis reumatoide (AR) la afectación cardiovascular se ha descrito como una de las primeras causas de mortalidad $^{8,9}$, independiente de la prevalencia de factores de riesgo cardiovascular clásicos en este grupo de pacientes ${ }^{10}$. Por otro lado, podríamos considerar que la edad avanzada de nuestros pacientes puede deberse a la mayor supervivencia de los pacientes con EAS, si bien Petri ${ }^{11}$ destaca que la supervivencia a 10 años en los pacientes con LES no ha variado desde la década de 1980. En ese mismo artículo, no obstante, se refleja que la mortalidad a 15 años supera $60 \%$.

Los pacientes con EAS pueden desarrollar afectación cardiaca a cualquier nivel, y sus manifestaciones varían de leves o incluso subclínicas, hasta graves o potencialmente mortales ${ }^{13}$. La base etiopatogénica fundamental de la afectación cardiovascular parece residir en la inflamación crónica subyacente en las EAS, por un proceso inmunomediado del sistema vascular ${ }^{14}$, en la que el estado de inflamación crónica y la disregulación del sistema inmune puede desencadenar, en primer lugar, disfunción endotelial subclínica, y posteriormente el desarrollo del proceso de aterogénesis ${ }^{15}$. Se han implicado numerosas moléculas en la cascada inflamatoria relacionada con la aterosclerosis, entre las que destacan los reactantes de fase aguda (especialmente proteína
C reactiva), las citoquinas proinflamatorias (como el factor de necrosis tumoral alfa y las interleukinas 6 y 18), las moléculas de adhesión (como VCAM-1 e ICAM-1), y factores específicos (como los anticuerpos anticardiolipinas ${ }^{16}$, así como el aumento del estrés oxidativo y la reducida disponibilidad de óxido nítrico endotelial ${ }^{17}$. Por todo ello, además de los marcadores ya conocidos de aterosclerosis, se están investigando otros nuevos en los últimos años, como la dimetil arginina asimétrica $(\mathrm{ADMA})^{17}$, si bien a día de hoy no existe ningún marcador bioquímico eficaz que detecte lesión endotelial precoz, por lo que se sigue proponiendo como primera actitud más eficaz en estos pacientes, el control estricto de los FRCV convencionales ${ }^{14} \mathrm{y}$ el estudio cardiovascular con todas las herramientas diagnósticas de las que se disponen a día de hoy, para detectar la afectación preclínica y mejorar el pronóstico de estos pacientes a largo plazo $^{17}$.

Además, el estado protrombótico inherente a algunas EAS (por ejemplo el síndrome antifosfolípido), y las propias intervenciones terapéuticas, pueden aumentar aún más el estado de ateromatosis acelerada que tiene lugar en los pacientes con $\mathrm{EAS}^{14}$.

Nuestro estudio tiene varias limitaciones. En primer lugar, no se han incluido pacientes que acuden al SU por motivos dermatológicos, oftalmológicos o traumatológicos, que consultan en otra unidad de urgencias en nuestro Hospital. En segundo lugar, los pacientes que acuden directamente a consultas del médico especialista ante cualquier urgencia no vital, no están recogidos en este estudio, aunque este tipo de consulta no correspondería a una descompensación aguda urgente o emergente.

En nuestro estudio hemos encontrado una prevalencia de $0,91 \%$ de consultas de pacientes con EAS que acuden al SU. Esto no es comparable con otros estudios que han analizado las consultas de causa reumatológica en los Servicios de Urgencias en la población general y no las causas que motivan una consulta en Urgencias de los pacientes con diagnóstico de EAS. En este sentido, Schlosser et $\mathrm{al}^{3}$, describe $9 \%$ de consultas de causa reumatológica de la población general en un período de dos meses en urgencias, de las cuales $83 \%$ se diagnosticaron durante el seguimiento de una condición de causa reumatológica.

El perfil de pacientes con EAS que consulta 
en nuestro Servicio de Urgencias, es una mujer, con diagnóstico previo de $\mathrm{AR}$, mayor de 70 años, que consulta más frecuentemente por motivos cardiovasculares $(25 \%$ de los pacientes con EAS comparado con $6,5 \%$ de la población general) y que requieren ingreso hospitalario con una frecuencia mayor que la población general $(47,6 \%$ en este grupo de pacientes con EAS comparado con $8-9 \%$ de ingresos del Servicio de Urgencias General).

Por todo ello, creemos necesario desarrollar estudios con un período de observación más amplio, mayor número de pacientes que permita evaluar la verdadera prevalencia de los pacientes con EAS que acuden a los $\mathrm{SU}$, sus principales motivos de consulta y el pronóstico de las complicaciones urgentes que presenten. Esto permitirá mejorar la atención médica de estos pacientes en urgencias y ayudará a los distintos especialistas en el ámbito ambulatorio a realizar estudios precoces de prevención de las principales causas de morbimortalidad. Más allá del control de la enfermedad de base y sus complicaciones, el control de la inflamación crónica, la ateromatosis acelerada y el estricto control de los factores de riesgo cardiovascular son cruciales en este subgrupo de pacientes.

Del mismo modo, es un desafío el desarrollo de nuevos estudios que definan biomarcadores precoces en el proceso de aterosclerosis acelerada y prematura de estos pacientes, estrategias de cuantificación del verdadero riesgo cardiovascular, así como, herramientas más eficientes en el diagnóstico precoz de lesión endotelial, pues en el conjunto de las complicaciones de las EAS, esta representa una carga importante de morbimortalidad.

\section{Referencias}

1. Vecina ST, Feixas MS. Urgencias en las enfermedades autoinmunes del tejido conectivo. Emergencias 2003; 15: $175-82$.

2. Slobodin G, Hussein A, Rozenbaum M, Rosner I. The emergency room in systemic rheumatic diseases. Emerg Med J 2006; 23 (9): 667-71.

3. Schlosser G. An analysis of rheumatology cases presenting to the emergency room of a teaching hospital. J Rheumatol 1988; 15: 356-8.

4. Rojas-Serrano J, Cardiel MH. Lupus patients in an emergency unit. Causes of consultation, hospitalization and outcome. A cohort study. Lupus 2000 (9); 601-6.

5. Panopalis P, Gillis JZ, Yazdany J, Hersh A, Julian L, Criswell LA, et al. Frequent use of the emergency department among persons with systemic lupus erythematosus. Arthritis Care Res (Hoboken) 2010; 62 (3): 401-8.

6. Smith EC, Berry H, Scott L. The clinical need for an acute rheumatology referral service. British Lournal of Rheumatology 1996; 35: 389-91.

7. Urowitz MB, Bookman AA, Koehler BE, Gordon DA, Smythe HA, Ogryzlo MA. The bimodal mortality pattern of systemic lupus erythematous. Am J Med 1976; 60: $1-5$.

8. Solomon D, Karlson EW, Rimm EB, Cannuscio CC, Mandl LA, Manson JE, et al. Cardiovascular morbidity and mortality in women diagnosed with rheumatoid arthritis. Circulation 2003; 107: 1303-7.

9. Aviña-Zubieta JA, Choi HK, Sadatsafavi M, Etminan M, Esdaile JM, Lacaille D. Risk of cardiovascular mortality in patients with rheumatoid arthritis: a metaannalysis of observational studies. Arthritis Rheum 2008; 59: 1690-7.

10. Del Rincón ID, Williams K, Stern MP, Freeman GL, Ecalante A. High incidence of cardiovascular events in a rheumatoid arthritis cohort not explained by traditional cardiac risk factors. Artrhitis Rheum 2001; 44: 2737-45.

11. Petri M. Life-Threatening complications of Systemic Lupus Erythematosus. M.A. Khamashta and M. Ramos-Casals (eds.), Autoimmune Diseases, 9. DOI: 10.1007/978-0-85729-358-9_2, (C) Springer-Verlag London Limited 2011.

12. Ward MM. Hospital experience and mortality in patients with systemic lupus erythematous: which patients benefit most from treatment at highly experienced hospitals? J Rheumotol 2002; 29 (6): 1198-206.

13. Villa-Forte A, Mandell BF. Cardiovascular Disorders and rheumatic disease. Rev Esp Cardiol 2011; 64 (9): 809-17.

14. Shoenfeld Y, Gerli R, Doria A, Matsuura E, Cerinic $\mathrm{MM}$, Ronda N, et al. Accelerated atherosclerosis in Autoimmune Rheumatic Diseases. Circulation 2005; 112: 3337-47.

15. Sitia S, Tomasoni L, Atzeni F, Ambrosio G, Cordiano C, Catapano A, et al. From endothelial dysfunction to atherosclerosis. Autoimmunity Reviews 2010; 9: 830-4.

16. Balanescu S, Calmac L, Constantinescu D, Marinescu M, Onut R, Dorobantu M. Systemic inflammation and early atheroma formation: are they related? Maedica (Buchar) 2010; 5 (4): 292-301.

17. Sitia S, Gianturco L, Turiel M. Role of cardiovascular imaging in systemic autoimmune diseases. World J Cardiol 2010; 2 (8): 237-42. 